\title{
Time scaling with efficient time-propagation techniques for atoms and molecules in pulsed radiation fields
}

\author{
Aliou Hamido, ${ }^{1}$ Johannes Eiglsperger, ${ }^{2,3}$ Javier Madroñero, ${ }^{3}$ Francisca Mota-Furtado, ${ }^{4}$ Patrick O’Mahony, ${ }^{4}$ \\ Ana Laura Frapiccini, ${ }^{1, *}$ and Bernard Piraux ${ }^{1}$ \\ ${ }^{1}$ Institute of Condensed Matter and Nanosciences, Université Catholique de Louvain, Bâtiment de Hemptinne, 2, chemin du cyclotron, \\ B-1348 Louvain-la Neuve, Belgium \\ ${ }^{2}$ Institut für Theoretische Physik, Universität Regensburg, D-93040 Regensburg, Germany \\ ${ }^{3}$ Physik Departement, Technische Universität München, D-85747 Garching, Germany \\ ${ }^{4}$ Department of Mathematics, Royal Holloway, University of London, Egham, TW20 OEX Surrey, United Kingdom
}

(Received 6 May 2011; published 27 July 2011)

\begin{abstract}
We present an ab initio approach to solving the time-dependent Schrödinger equation to treat electron- and photon-impact multiple ionization of atoms or molecules. It combines the already known time-scaled coordinate method with a high-order time propagator based on a predictor-corrector scheme. In order to exploit in an optimal way the main advantage of the time-scaled coordinate method, namely, that the scaled wave packet stays confined and evolves smoothly toward a stationary state, of which the squared modulus is directly proportional to the electron energy spectra in each ionization channel, we show that the scaled bound states should be subtracted from the total scaled wave packet. In addition, our detailed investigations suggest that multiresolution techniques like, for instance, wavelets are the most appropriate ones to represent the scaled wave packet spatially. The approach is illustrated in the case of the interaction of a one-dimensional model atom as well as atomic hydrogen with a strong oscillating field.
\end{abstract}

DOI: $10.1103 /$ PhysRevA.84.013422

PACS number(s): $32.80 . \mathrm{Rm}$

\section{INTRODUCTION}

During the last few years, substantial progress regarding the development of new extreme ultraviolet sources has been made in two directions. On the one hand, high-order harmonic generation has been used to produce attosecond pulses with a duration of the order of the characteristic time scale of the inner-shell electron dynamics in atoms and molecules [1]. On the other hand, free-electron lasers [2] are now operating at unprecedentedly high peak intensities in the far-x-ray regime. These developments have opened the route to the exploration of nonlinear processes in the short-wavelength limit. At present, processes such as multiphoton multiple ionization of atoms and molecules are the focus of many experimental and theoretical studies with a view to understanding the subtle role of the electronic correlations.

Within this context, there is clearly a need for reliable theoretical and numerical methods that provide accurate solutions of the time-dependent Schrödinger equation (TDSE). To this end, however, it is necessary to overcome the following four main difficulties. (i) The continuum components of the wave packet expand in a rapidly increasing volume of space, thereby requiring very extended spatial grids or basis functions in order to avoid artificial reflections from the numerical boundaries. (ii) Increasingly large spatial phase gradients develop within the wave packet with time, demanding very dense grids or large basis sizes. (iii) Solving the TDSE on a spatial grid or in a basis of square integrable functions leads to a stiff system of equations which, in principle, makes explicit time propagators unstable. Finally, (iv) the direct extraction

\footnotetext{
* On leave from División Colisiones Atómicas, Centro Atómico Bariloche, 8400 S. C. de Bariloche, Rio Negro, Argentina.
}

of information on the multielectron continua from the wave packet necessitates knowledge of the asymptotic behavior of the corresponding wave function.

The existing time-dependent approaches have been mainly used to study single and double ionization of two-electron atoms and molecules by intense ultrashort radiation fields. In the low-frequency regime where the calculations are extremely challenging, Smyth et al. [3] have developed a fully numerical method to solve the TDSE. It has provided valuable qualitative information on the role of the electronic correlations and the so-called rescattering process [4]. In the high-frequency regime where one or two photons are involved in the ionization process, there are presently two types of treatment to solve the TDSE: treatments based on standard methods of collision theory and the close-coupling approaches. In the former case, the wave packet is time propagated on an extended spatial grid during a period of time that is much larger than the pulse duration. The Fourier transform of the wave packet provides a scattered wave function which is then analyzed by means of time-independent methods. Palacios et al. [5] use the exterior complex scaling technique, which maps an outgoing wave into a vanishing wave outside a physically unaltered region, allowing the extraction of the relevant information on the various ionization processes without the necessity of knowing the asymptotic behavior of the wave function associated with the multiple continua. Recently, Malegat et al. [6] applied the method of the hyperspherical $R$ matrix with semiclassical outgoing waves to calculate the various ionization yields. In this method, the scattered wave is propagated semiclassically with respect to the hyperradius, all the way to the asymptotic region where the various ionization channels are decoupled.

Many approaches based on the close-coupling method have been developed. They essentially differ in the way the information on the ionization processes is extracted from the 
wave packet. The most common way is to propagate the wave packet freely after the interaction with the pulse, until it reaches a spatial region where the ionization channels are assumed to be decoupled. It is then projected onto an uncorrelated product of Coulomb functions in each of the ionization channels [7-10]. Instead of using uncorrelated products of Coulomb functions, Ivanov and Kheifets [11] project the wave packet onto continuum-state wave functions obtained by means of the convergent-close-coupling method, which takes electron correlations into account in an approximate way. A different procedure has been developed by Foumouo et al. [12]. Since the asymptotic behavior of the single continuum wave function is known, it is convenient to calculate the total probability for double ionization by subtracting the total probability for single ionization from the all-inclusive probability for breakup, which in turn can be calculated without any reference to the boundary conditions. In order to calculate the total and partial probabilities for single ionization, Foumouo et al. use the Jacobi-matrix method to generate a multichannel scattering wave function that describes the single continuum. The projection of the wave packet onto this scattering wave function is performed just at the end of the interaction of the two-electron system with the radiation pulse.

Finally, Lysaght et al. [13,14] have recently initiated the development of a time-dependent $R$-matrix approach to describe complex multielectron atoms and atomic ions in intense ultrashort radiation pulses. This approach consists in time-propagating the atomic wave function in the presence of the radiation field in both the internal and external $R$-matrix regions.

The present approach combines the time-scaled coordinate (TSC) method with a high-order fully implicit predictorcorrector scheme for the time propagation. The timedependent scaling of the radial electronic coordinates together with a phase transformation of the wave packet allow for "freezing" the spatial expansion of the wave packet in this representation while removing fast oscillations due to the increasingly large spatial phase gradients that develop with time. This method is in fact equivalent to using a time-dependent basis that expands in the same way as the wave packet itself. This idea of time-scaling the coordinates is not new and has been widely exploited in many different fields of physics. In 1979, Burgan et al. [15] studied the Schrödinger equation for a multidimensional quantum harmonic oscillator with time-dependent frequencies. By introducing an appropriate time-dependent scaling of the spatial coordinates, they were able to transform the problem to a free-particle motion and to derive an exact analytical solution. Later on, Manfredi et al. [16,17] introduced a time-dependent scaling of both space and time variables to freeze the expansion into a vacuum of both a one-dimensional, collisionless, two-species classical plasma and a quantum electron gas in planar geometry. In atomic and molecular physics, Solov'ev and Vinitsky [18] and later on, Ovchinnikov et al. [19] treated the Coulomb three-body problem, in particular ion-atom and atom-atom collisions, within a proper adiabatic representation by timescaling the internuclear distance. More recently, the TSC method has been used by Sidky and Esry [20] and Derbov et al. [21] to treat the interaction of a model atom and molecule with an electromagnetic pulse, and by Serov et al.
$[22,23]$ to study electron-impact single and double ionization of helium and later double photoionization of two-electron atomic systems [24]. The TSC method is an extension of a self-similarity analysis which has been introduced recently in astrophysics [25]. By an appropriate scaling of all variables entering the equations governing the dynamics of a very large hydrodynamic system, the rescaled equations are identical to the original ones. This allows the definition of dual equivalent systems, the first one characterized by very long time and parsec-length scales and the second one by very short time and small length scales, allowing its study at the laboratory scale. Finally, the TSC method has been used to study the expansion of a Bose-Einstein condensate following the switch-off of the trap [26,27].

In the case of the interaction of an atom or a molecule with an electromagnetic pulse, the TSC method effectively confines the expansion of the scaled wave packet within a finite space of controllable size so that the evolution of this scaled wave packet can be followed over very long periods of time. Furthermore, it has been shown [21,22,28] that a long time after the end of the interaction of the atom or the molecule with the pulse, the energy spectrum of the ejected electrons is simply proportional to the squared modulus of this scaled wave packet. The confinement of the scaled wave packet is due to three factors: the presence of a harmonic potential, the narrowing of the atomic potential, and the increase of the effective mass of the electrons with time. This means that the effective de Broglie wavelength of these electrons decreases. In other words, the TSC method introduces different length scales into the problem. This has two important consequences. First, an optimal spatial description of the scaled wave packet requires multiresolution techniques, and second, it increases significantly the stiffness of the system of first-order differential equations that must be solved for the time propagation of the wave packet. In other words, the time step rapidly decreases with increasing size of the system [29]. In this paper, we describe a seventh-order fully implicit predictor-corrector scheme. The predictor is the fifth-order explicit method of Fatunla [30,31] while the corrector is a seventh-order fully implicit Radau method [34]. In principle, an implicit scheme requires solving large systems of algebraic equations at each time step. However, the accuracy of Fatunla's method is high enough $[32,33]$ to allow the use of an iterative procedure, the biconjugate gradient algorithm, to solve the large systems of algebraic equations at the corrector level. In other words, only matrix-vector products are needed, allowing a deep parallelization of the computer code.

This paper is organized as follows. In the next section, we describe the TSC method in detail. For the sake of illustration, we consider the interaction of a one-dimensional system modeled by a Gaussian potential and interacting with a cosine-squared electromagnetic pulse. First, we examine the different reasons for the confinement of the scaled wave packet. Then, we study various spatial representations of this scaled wave packet and study its behavior at various times after the pulse has ceased to interact with the model atom. In the next section, we describe our time propagation method. We first start with the explicit Fatunla method and then discuss in detail the predictor-corrector scheme. The fourth section is devoted to the calculation of the energy spectrum. We derive 
an analytical expression in the case of our model atom and for atomic hydrogen. Results for both cases are presented and discussed in detail. The last section is devoted to conclusions and perspectives. Unless stated otherwise, atomic units are used throughout this paper.

\section{THE TIME-SCALED COORDINATE METHOD}

\section{A. Outline of the method}

Our one-dimensional model that serves as an illustration for describing the TSC method consists of an electron initially bound in a Gaussian potential and interacting with a cosinesquared electromagnetic pulse. The TDSE that governs the dynamics of the electron is

$$
i \frac{\partial}{\partial t} \Psi(x, t)=\left[H_{0}(x)+H_{I}(x, t)\right] \Psi(x, t) .
$$

The atomic Hamiltonian $H_{0}(x)$ is given by

$$
H_{0}(x, t)=-\frac{1}{2} \frac{\partial^{2}}{\partial x^{2}}+V(x)
$$

where

$$
V(x)=-V_{0} e^{-\beta x^{2}} .
$$

By adjusting the parameters $V_{0}$ and $\beta$ that fix the depth and the width of the Gaussian potential, we can easily vary the number of bound states. In all the calculations we perform, we always assume that the model atom is initially in its ground state. Within the dipole approximation and in the velocity form, the interaction Hamiltonian $H_{I}(x, t)$ is written

$$
H_{I}(x, t)=-i A_{0} f(t) \sin (\omega t+\varphi) \frac{\partial}{\partial x} .
$$

$A_{0}$ is the amplitude of the vector potential which is polarized along the $x$ axis. $\omega$ is the frequency and $\varphi$ the carrier phase. $f(t)$ is the pulse envelope, defined as follows:

$$
f(t)= \begin{cases}\cos ^{2}\left(\frac{\pi}{\tau} t\right), & |t| \leqslant \frac{\tau}{2}, \\ 0, & |t|>\frac{\tau}{2} .\end{cases}
$$

The total pulse duration $\tau=2 \pi n_{\mathrm{c}} / \omega$ where $n_{\mathrm{c}}$ is an integer giving the number of optical cycles. The fact that $n_{\mathrm{c}}$ is an integer is important since it ensures that the electric field has no static components.

According to the TSC method [20], we introduce the scaled coordinate $\xi$ given by

$$
\xi=\frac{x}{R(\sigma)},
$$

where $R(\sigma)$ is an arbitrary scaling function. It is important to stress that $\sigma$ is just a parameter. In the following, we assume that it coincides with the time $t$. We write the scaled wave packet as follows:

$$
\Phi(\xi, t)=\sqrt{R} e^{-i R \dot{R} \xi^{2}} \Psi(R \xi, t),
$$

where the overdot indicates the time derivative. The factor $\sqrt{R}$ ensures that this scaled wave packet is correctly normalized. The phase transformation absorbs the fast oscillations of the unscaled wave packet during its time evolution. The scaled wave packet $\Phi(\xi, t)$ satisfies the following TDSE:

$$
\begin{aligned}
i \frac{\partial}{\partial t} \Phi(\xi, t)= & {\left[-\frac{1}{2} \frac{\partial^{2}}{R^{2} \partial \xi^{2}}+V(R \xi)\right.} \\
& \left.-i \frac{A_{0}}{R} f(t) \sin (\omega t+\varphi) \frac{\partial}{\partial \xi}+\frac{1}{2} R \ddot{R} \xi^{2}\right] \Phi(\xi, t) .
\end{aligned}
$$

Since the idea behind the TSC method is to build a timedependent basis that expands in the same way as the wave packet, it is expected that, if this expansion is accelerated, noninertial forces should appear. This explains the presence of the harmonic potential in the above TDSE. When this expansion occurs at constant velocity, i.e. when the scaling function is linear with time, the harmonic potential disappears. For $\ddot{R}>0$, this potential confines the wave packet in a finite space. In fact, in the absence of external fields, the spectrum of the operator in square brackets in Eq. (8) becomes purely discrete [23]. An analysis of Eq. (8) shows that the scaling transformation (6) introduces an electron effective mass that is proportional to $R^{2}$. Finally, we see that, when $R$ increases, the Gaussian potential narrows with time. This shrinking mainly affects the bound-state components of the wave packet. Note that in the case of a Coulomb potential it is the effective electric charge that goes to zero with increasing values of $R$.

Before analyzing in more detail the different factors that lead to the confinement of the scaled wave packet, let us examine the scaling function $R(t)$. As stressed in [20], this function is arbitrary and chosen to facilitate the numerics. It must, however, satisfy a few constraints. It should be real, larger than 1 , and equal to 1 from $t=t_{\text {initial }}$ corresponding to the beginning of the interaction until $t=t_{\mathrm{sc}}$ where the scaling starts. In addition, for large time $t, R$ should tend to $R_{\infty} t$ where $R_{\infty}$ is what we call the asymptotic velocity. This ensures that for large times the scaled wave packet becomes stationary. In the present calculations, we define $R(t)$ as follows:

$$
R(t)=\left\{\begin{array}{cl}
1, & t \leqslant t_{\mathrm{sc}} \\
\left\{1+\left[R_{\infty}\left(t-t_{\mathrm{sc}}\right)\right]^{n}\right\}^{1 / n}, & t>t_{\mathrm{sc}} .
\end{array}\right.
$$

This form with $n=4$ has been used by several authors $[20,21,28]$. It leads to a smooth transition to the linear regime where the harmonic potential is switched off. The scaling function for $n=3, t_{\mathrm{sc}}=t_{\text {initial }}$, and $R_{\infty}=0.025$ together with a sine-squared pulse envelope are shown as functions of time in Fig. 1. In the shaded region, between 10 and 60 a.u. of time, $\ddot{R}(t)$ is significantly larger than zero. In that case, the confinement of the scaled wave packet results predominantly from the presence of the harmonic potential. For times $t>60$, the harmonic potential is smoothly switched off but the confinement of the scaled wave packet subsists because of the electron effective mass, which rapidly increases. As soon as the scaling starts (at $t=t_{\mathrm{sc}}$ ), the atomic bound-state wave functions start to shrink because of the narrowing of the atomic potential. In the case of the Gaussian potential it is easy to show that the width is inversely proportional to $R$. This is illustrated in Fig. 2, where we show the scaled ground-state wave function as well as the effective potential, which is the sum of the scaled Gaussian potential and the harmonic one, 
as functions of the scaled variable $\xi$, for three different values of $t: t_{\mathrm{sc}}, t_{\mathrm{sc}}+15$ a.u., and $t_{\mathrm{sc}}+80$ a.u. Note that at $t=t_{\mathrm{sc}}$, the effective potential reduces to the Gaussian potential. The fact that the scaled ground-state wave function shrinks rapidly is due to the relatively high value of the asymptotic velocity which, in the present case, is equal to 0.1 a.u. As $n=4$ [see Eq. (9)], $t=t_{\mathrm{sc}}+80$ a.u. corresponds to a time for which the scaling function has reached its linear regime. It is important to mention that the results given in Fig. 2 require only a partial diagonalization of the atomic Hamiltonian and no time propagation. Therefore, they provide an easy way to control the fineness of the spatial discretization necessary to maintain the accuracy of the time propagation.

\section{B. Spatial representation of the scaled wave packet}

The optimal way of describing the wave packet in space is based on a multiresolution analysis [35]. The general idea is to define different resolution levels in various regions of space through the introduction of several grids with a density of mesh points that increases from one grid to the next in the spatial regions of interest. These techniques will be analysed in detail in a forthcoming publication. Here, we use two different spectral methods. The first one consists in developing the wave

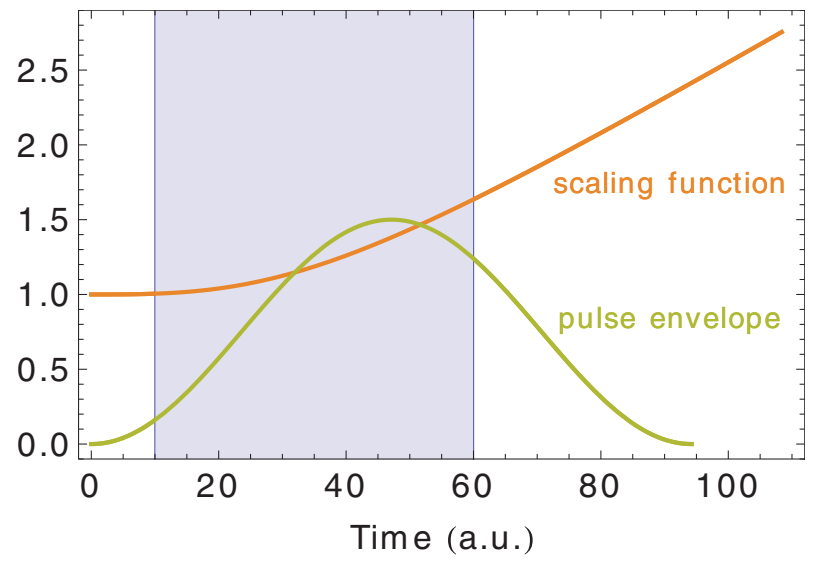

FIG. 1. (Color online) Scaling function and pulse envelope as functions of time. For the scaling function, $n=3, t_{\mathrm{sc}}=t_{\text {initial }}$, and $R_{\infty}=0.025$. The pulse has a sine-squared envelope and a total duration of 94 a.u.

packet on $\mathcal{L}^{2}$-integrable functions, namely, Hermite-Sturmian functions in the case of our one-dimensional model and Coulomb-Sturmian functions in the case of atomic hydrogen. The second method uses a basis of $B$ splines built on a
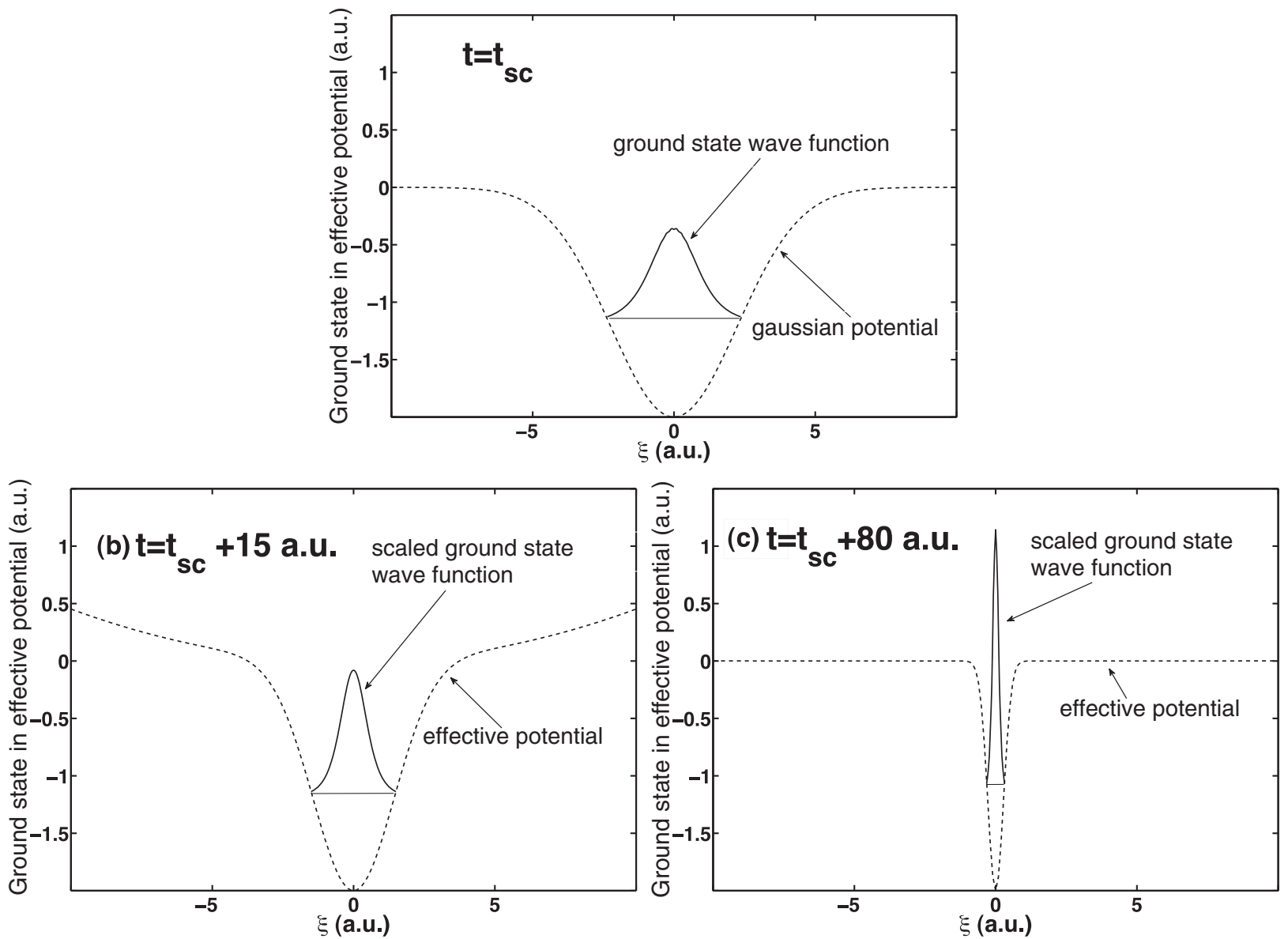

FIG. 2. Effective potential, namely, the sum of the scaled Gaussian potential and the harmonic one, together with the scaled ground-state wave function versus the scaled variable $\xi$. Three times are considered: (a) $t=t_{\mathrm{sc}}$ corresponding to the time where scaling is switched on, (b) $t=t_{\mathrm{sc}}+15$ a.u., and (c) $t=t_{\mathrm{sc}}+80$ a.u. The asymptotic velocity is equal to 0.1 a.u. and $n=4$ [see Eq. (9)]. 
nonuniform grid with an exponential sequence of break points. These two methods, which are far from being optimal, have the merits of being easily implemented and of showing clearly that one cannot dissociate the spatial discretization problem from the time propagation.

Let us now briefly describe our first spectral method. In the case of our one-dimensional model, we expand the total wave packet in a finite basis of Hermite-Sturmian functions as follows:

$$
\Psi(x, t)=\sum_{n=0}^{N} a_{n}(t) \varphi_{n}^{\alpha}(x),
$$

where $a_{n}(t)$ is the expansion coefficient and $\varphi_{n}^{\alpha}(x)$ the HermiteSturmian function given by

$$
\varphi_{n}^{\alpha}(x)=\left(2^{n} n ! \sqrt{\frac{\pi}{\alpha}}\right)^{-1 / 2} e^{-(\alpha / 2) x^{2}} H_{n}(\sqrt{\alpha} x) .
$$

The elements of the matrices associated with all operators present in the scaled and unscaled Hamiltonians can be calculated analytically except for the Gaussian potential. In this latter case, a Gauss-Hermite quadrature provides exact results for a sufficient number of abscissas. $\alpha$ is a dilation parameter that determines the resolution of the basis. Indeed, a large value of $\alpha$ gives a good description of a wave packet which exhibits sharp variations close to the origin. In that case, however, a large value of $N$, the number of basis functions, is necessary if the extent of this wave packet is significant. By contrast, a small value of $\alpha$ allows a good description of the wave packet over much larger distances but $N$ has to be extremely large again if sharp variations of the wave packet occur.

In the case of atomic hydrogen, where we use spherical coordinates, we write the wave packet as follows:

$$
\Psi(\vec{r}, t)=\sum_{n, l, m} a_{n, l}(t) \frac{S_{n, l}^{\kappa}(r)}{r} Y_{l, m}(\theta, \phi),
$$

where $Y_{l, m}(\theta, \phi)$ is a spherical harmonic and $S_{n, l}^{\kappa}(r)$ the Coulomb-Sturmian function given by

$$
S_{n, l}^{\kappa}(r)=N_{n, l}^{\kappa} r^{l+1} e^{-\kappa r} L_{n-l-1}^{2 l+1}(2 \kappa r) .
$$

$N_{n, l}^{\kappa}$ is a normalization factor (see [12] for details) and $L_{n-l-1}^{2 l+1}(2 \kappa r)$ a Laguerre polynomial. The index $n$ varies from $l+1$ to $\infty$. The Coulomb Sturmian functions form a complete and discrete set of $\mathcal{L}^{2}$-integrable functions that are exact solutions of the stationary Schrödinger equation for a single electron in the Coulomb field of a nucleus of charge $Z$ for selected values of $\kappa$. As a result, these functions are well adapted to describe the energy spectrum of atomic hydrogen as well as its behavior in the presence of an external field. In fact, the matrices associated with the corresponding Hamiltonian are banded with a narrow bandwidth (three diagonals). As in the case of the Hermite-Sturmian functions, a given basis of Coulomb-Sturmian functions is characterized by a fixed value of the dilation parameter $\kappa$. An interesting idea in the spirit of the multiresolution approaches is to consider a set of different values of $\kappa$ within a given basis in order to take into account the various length scales in the problem. Despite the fact that the introduction of different values of $\kappa$ makes the basis numerically overcomplete, thereby requiring the elimination of the linearly dependent eigenvectors of the overlap matrix, this idea turned out to be extremely successful in generating the singly and doubly excited states of helium [12,36-39]. Note that, in the case of atomic hydrogen, the time-dependent scaling of the radial coordinate is equivalent to introducing a time-dependent $\kappa$.

In our second method to treat our one-dimensional model, we expand the wave packet in a basis of $B$ splines [40]:

$$
\Psi(x, t)=\sum_{i=1}^{N} c_{i}(t) B_{i}^{k}(x),
$$

where $B_{i}^{k}(x)$ is a $B$ spline of order $k$. In the present calculations, $k=7$. In order to calculate the $B$ splines, we use an exponential sequence of break points. In practice, we proceed as follows. We adjust the asymptotic velocity and the time $t_{\mathrm{sc}}$ at which the scaling starts so that the scaled wave packet is confined in a relatively small interval $\left[-x_{\max },+x_{\max }\right]$. We then define two symmetrical exponential sequences of break points in the intervals $\left[-x_{\max }, 0\right]$ and $\left[0,+x_{\max }\right]$. In the interval $\left[0,+x_{\max }\right]$, for instance, the break points $\xi_{i}$ are given by

$$
\xi_{i}=x_{\max }\left(\frac{e^{\gamma[(i-1) /(N-1)]}-1}{e^{\gamma}-1}\right), \quad i=1, \ldots, N .
$$

Typically, we have $x_{\max }=35, \gamma=5$, and $N$ of the order of 100 . In this case, break points accumulate symmetrically around 0 in order to describe properly the shrinking of the bound states. However, this accumulation of break points introduces high frequencies in the problem. In this $B$-spline basis, the energy spectrum of the unscaled atomic Hamiltonian contains very high positive eigenenergies of the order of 3000 a.u. If the wave packet is time propagated in the $B$-spline basis, each component of the $B$ splines contains these high frequencies, making the problem extremely stiff and leading to a dramatic decrease of the time step. Note that this difficulty can be avoided by propagating the scaled wave packet in the atomic basis, i.e., the basis in which the unscaled atomic Hamiltonian is diagonal. In that case, however, it is necessary to solve a generalized eigenvalue problem, which could be time consuming in the case of a more complex system like He or $\mathrm{H}_{2}$.

\section{Evolution of the scaled wave packet}

Before examining the time evolution of scaled wave packets, it is instructive to analyze the behavior of the unscaled ones. We first consider the case of a Gaussian potential with $V_{0}=1$ a.u. and $\beta=1$ a.u. This potential has only one bound state, the energy of which is equal to -0.477 a.u. This model atom interacts with a cosine-squared laser pulse of peak intensity $I_{\text {peak }}=10^{13} \mathrm{~W} / \mathrm{cm}^{2}$ and frequency $\omega=0.7 \mathrm{a}$.u. The total duration of the pulse is six optical cycles. In Fig. 3, we show the real part of the wave packet at time $t=t_{\text {final }}$, i.e., at the end of the pulse (dark gray curve) and 300 a.u. of time later (light gray curve). We actually represent the ionized wave packet, i.e., the total wave packet without its bound-state component.

We clearly see that at time $t=t_{\text {final }}+300$ a.u., the real part of the ionized wave packet exhibits a much larger number of 


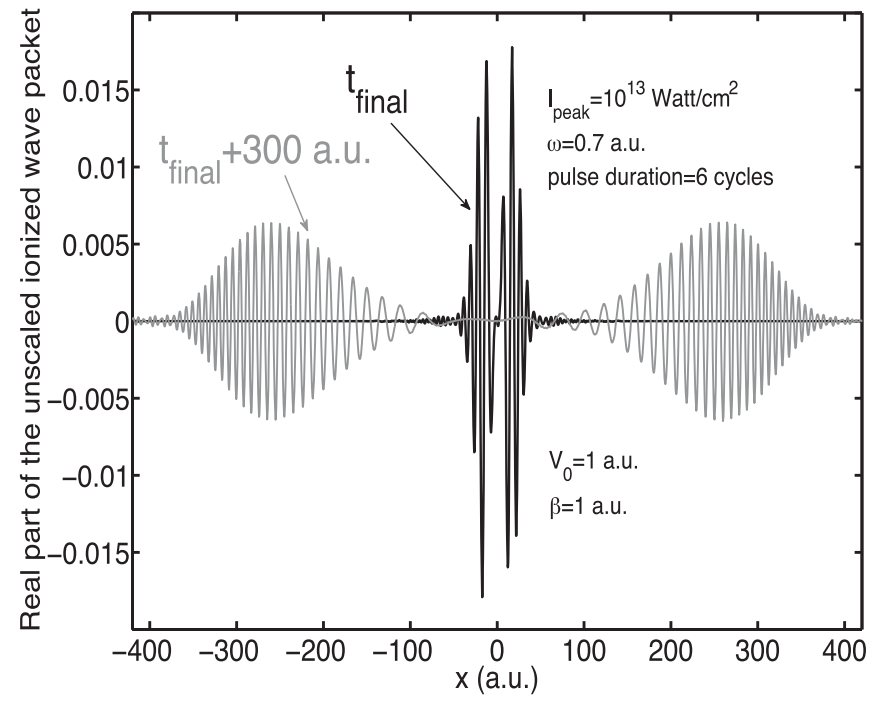

FIG. 3. Real part of the unscaled ionized wave packet resulting from the interaction of our one-dimensional model atom with a cosine-squared pulse of $10^{13} \mathrm{~W} / \mathrm{cm}^{2}$ peak intensity and 0.7 a.u. photon energy. The total duration of the pulse is six optical cycles. The Gaussian potential depth $V_{0}=1$ a.u. and $\beta=1$ a.u. The real part of the ionized wave packet is shown at time $t=t_{\text {final }}$, i.e., at the end of the pulse (dark gray curve) and at time $t=t_{\text {final }}+300$ a.u. (light gray curve).

oscillations than at $t=t_{\text {final }}$, the end of the pulse. In particular, we see the presence of a chirp for $t=t_{\text {final }}+300$. As stressed by Sidky and Esry [20], this results from the phase gradients that rapidly develop over large distances or, in other words, from the fact that the front edge of the wave packet is moving faster than the inner part. Let $\Psi\left(x, t_{\text {final }}\right)$ be the wave packet created at the end of the pulse. At any later time $t$, we have

$$
\Psi(x, t)=\int_{-\infty}^{\infty} d x^{\prime} \mathcal{G}\left(x, x^{\prime}, t\right) \Psi\left(x^{\prime}, t_{\text {final }}\right),
$$

where the Green function $\mathcal{G}\left(x, x^{\prime}, t\right)$ for a free electron is given by

$$
\mathcal{G}\left(x, x^{\prime}, t\right)=\sqrt{\frac{1}{2 i \pi t}} e^{i\left(x-x^{\prime}\right)^{2} / 2 t} .
$$

This means that the phase increases quadratically with the distance. In the present calculations, we need to use a basis of 1000 Hermite-Sturmian functions of parameter $\alpha=0.01$ to accurately reproduce all the oscillations over about 300 a.u. around the origin. In Fig. 4(a), we compare for $t=t_{\text {final }}+$ 250 a.u. the real parts of both the scaled and unscaled ionized wave packets for the same pulse and Gaussian potential parameters as in Fig. 3. In this particular case, the scaled ionized wave packet is obtained as follows. The unscaled wave packet is first propagated until $t=t_{\text {final }}$, the end of the interaction of our model atom with the pulse. At time $t=t_{\text {final }}$, the bound-state contribution is subtracted from the total wave packet and scaling is switched on. At time $t=t_{\text {final }}+250$ a.u., we clearly see on Fig. 4(a) that the scaled wave packet represented as a function of the position $\xi$ is confined compared to the unscaled one. In addition, the number of oscillations is already reduced.
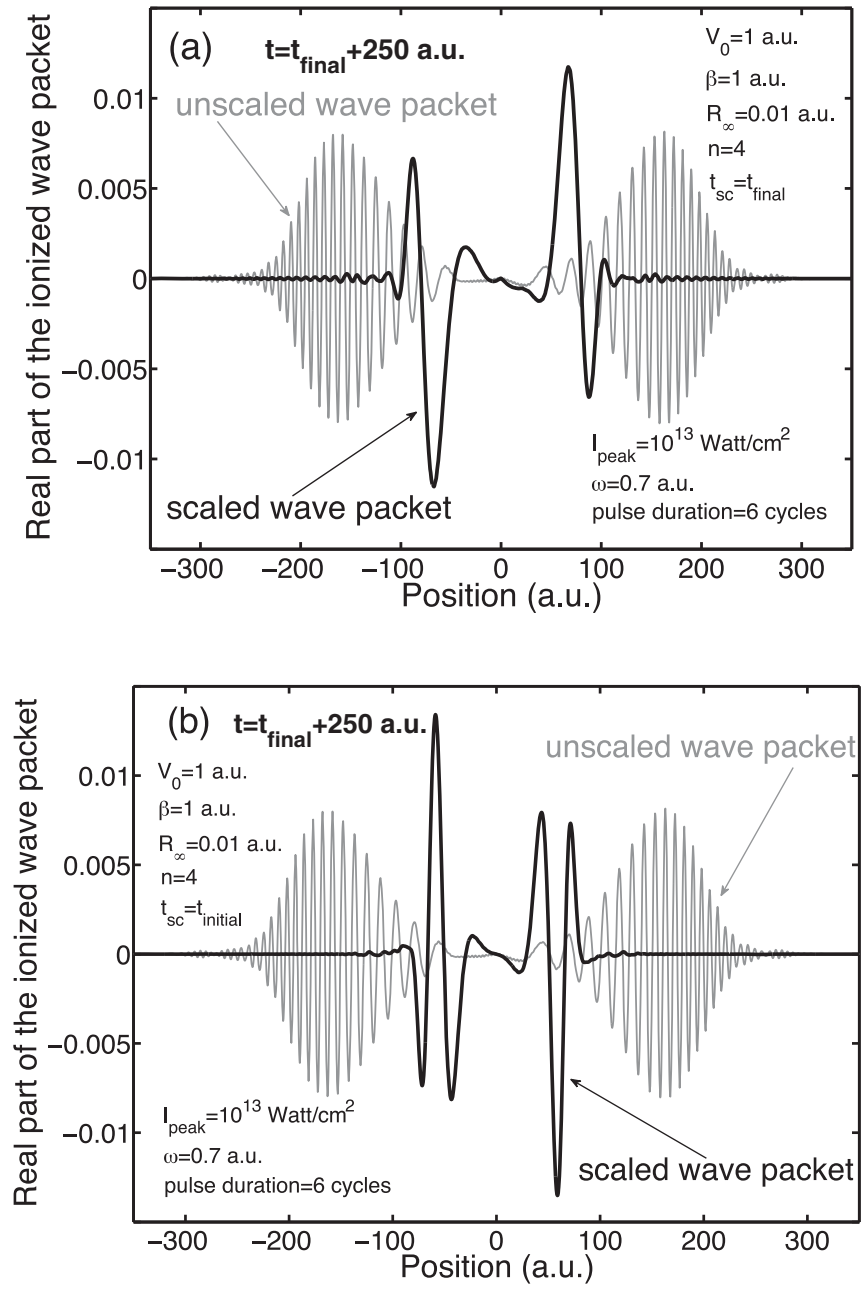

FIG. 4. Real part of both the scaled (dark gray curve) and unscaled (light gray curve) ionized wave packets resulting from the interaction of our one-dimensional model atom with the same pulse as in Fig. 3. The real parts of these wave packets are calculated at time $t=t_{\text {final }}+$ 250 a.u. and are represented as a function of the position: $x$ in the case of the unscaled wave packet and $\xi$ in the case of the scaled wave packet. The parameters of the Gaussian potential are the same as in Fig. 3. In the case of the scaled wave packet, the asymptotic velocity $R_{\infty}=0.01$ a.u., the parameter $n$ of the scaling function [see Eq. (9)] is equal to 4 , and the scaling is switched on (a) at the end of the interaction and (b) at the beginning of the interaction of our model atom with the pulse.

As discussed above, the quadratic increase of the phase with the distance is canceled by the phase transformation (7) of the wave packet. In principle, the time scaling of the coordinates may start at any time. In Fig. 4(b), we consider the same case as in Fig. 4(a) but the time scaling is now switched on right at the beginning of the interaction with the pulse. We clearly see that the confinement is slightly stronger and that the number of oscillations is significantly reduced. Indeed, beyond \pm 120 a.u., all the oscillations of weak amplitude present in Fig. 4(a) have disappeared. The comparison of Figs. 4(a) and 4(b) also shows that the scaled wave packet has not yet reached a stationary state. In Fig. 5, we show the scaled wave packet resulting from the interaction of the same one-dimensional 

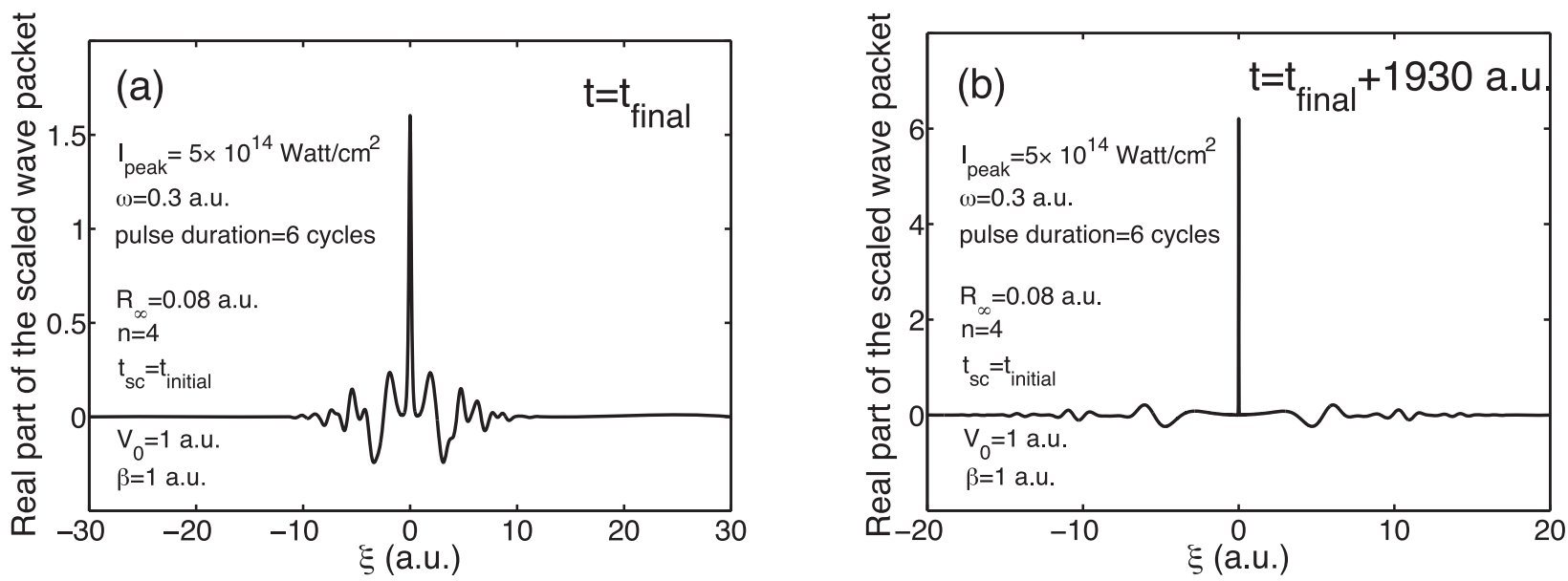

FIG. 5. Real part of the scaled wave packet resulting from the interaction of our one-dimensional model atom with a cosine-squared pulse of $5 \times 10^{14} \mathrm{~W} / \mathrm{cm}^{2}$ peak intensity and 0.3 a.u. photon energy. The total pulse duration is six optical cycles. The parameters of the Gaussian potential are the same as in Fig. 3. The time-dependent scaling is switched on at the beginning of the interaction with the pulse. The asymptotic velocity $R_{\infty}$ is equal to 0.08 a.u. while the parameter $n$ of the scaling function [see Eq. (9)] is equal to 4 . The real part of the scaled wave packet is represented as a function of $\xi$ at (a) $t=t_{\text {final }}$ and (b) $t=t_{\text {final }}+1930$ a.u.

model atom as before with a pulse of $5 \times 10^{14} \mathrm{~W} / \mathrm{cm}^{2}$ peak intensity and 0.3 a.u. photon energy. The total duration of the pulse is six optical cycles. In this particular case, we use $B$ splines to describe the scaled wave packet with two different exponential sequences of break points. The first grid with $\gamma=$ 5 [see Eq. (15)] and $200 B$ splines is used during the interaction of the model atom with the pulse. The second grid with $\gamma=$ 5 and $400 B$ splines is used after the interaction with the pulse, i.e., for $t>t_{\text {final }}$. In Fig. 5, we show the real part of the scaled wave packet at the end of the pulse [Fig. 5(a)] and 1930 a.u. of time later when the scaled wave packet becomes quasistationary [Fig. 5(b)]. The fast oscillations have now been completely removed. It is important to stress that, although the number of $B$ splines used is rather small, both grids allow us to describe accurately the shrinking of the ground state. In fact, for time $t \gg t_{\text {final }}$ when the scaling function becomes linear with time, the scaled TDSE is identical to the original TDSE within a simple linear scaling of the spatial coordinates. This clearly shows that a multiresolution approach in which the density of break points increases linearly with time around the origin makes sense. Note that, in the present case, the accumulation of break points around the origin (right from the beginning of the propagation) increases the stiffness of the system significantly, a problem which should be avoided by means of multiresolution techniques. The stiffness problem is discussed in the next section.

\section{TIME PROPAGATION}

Accurate solution of the TDSE usually requires the representation of the solution on large or/and dense grids or in large bases. In all cases, we deal with large systems of coupled first-order differential equations which are well known to be stiff [41]. This means that the step size decreases as the dimension of the system increases. The origin of the stiffness is clear: when the size or the density of the grid or the size of the basis is increased, the Hamiltonian generates large positive eigenenergies, which are responsible for strong oscillations in the solution of the TDSE. It is thus the largest positive eigenvalue which controls the step size. In fact, the stiffness of the system may lead to instability of the time propagation scheme as well as to inaccurate high-energy components of the solution [42]. Two approaches frequently used to overcome this problem are implicit schemes for solving the TDSE and the propagation of the TDSE in the atomic basis and, possibly, within the interaction picture. The first method requires typically the solution of large systems of linear equations at each integration step. It is important, however, to stress that implicit schemes actually solve the stability problem but not necessarily the inaccuracy problem for the high-energy components of the solution. In the second method, the time integration is achieved by means of explicit algorithms, which have been proved to be very stable for the solution of the TDSE in the atomic basis where the atomic Hamiltonian is diagonal. These algorithms need only matrix-vector products. However, the representation in the atomic basis requires the full diagonalization of the Hamiltonian before starting the integration. In any case, the computational cost increases dramatically with the size of the system.

It is therefore desirable to have an explicit algorithm suitable for the direct solution of stiff TDSEs. Such a method does exist and was proposed more than 30 years ago by Fatunla [30,31]. It has been successfully implemented for the description of single ionization of atoms by strong oscillating fields $[32,33]$. In this method, which takes into account the intrinsic frequencies of the system, the wave function is expressed in terms of oscillating functions. This leads to a simple recursive formula for the time propagation with a controlled error. At each integration step, only matrix-vector products are therefore needed. In the two following subsections, we describe the most important features of Fatunla's algorithm and its implementation and show how its accuracy can be significantly improved within a predictor-corrector scheme. 


\section{A. Fatunla's explicit scheme}

We start with the general matrix form of the TDSE using a spectral or a grid representation,

$$
i \mathbf{B} \frac{d \boldsymbol{\Psi}}{d t}=\mathbf{H}(t) \boldsymbol{\Psi},
$$

with $\mathbf{H}(t)$ the matrix representation of the Hamiltonian and $\mathbf{B}$ the overlap matrix, which is the identity in a grid representation or in an orthonormal basis. Truncation of the basis or of the grid leads to an $m$-dimensional first-order differential equation,

$$
\dot{\boldsymbol{\Psi}}=\boldsymbol{f}(t, \boldsymbol{\Psi}),
$$

where $\boldsymbol{f}(t, \boldsymbol{\Psi})=-i \mathbf{B}^{-1} \mathbf{H}(t) \boldsymbol{\Psi}$ is in general a complex $m$ dimensional function.

The stiffness of Eq. (19) leads to a solution $\Psi(t)$ which is an oscillating function. In a given interval $\left[t_{n}, t_{n+1}\right], t_{n+1}=$ $t_{n}+h$, with $h$ a small number, $\boldsymbol{\Psi}(t)$ is approximated by the function

$$
\boldsymbol{F}(t)=\left(\mathbf{I}-e^{\boldsymbol{\Omega}_{1} t}\right) \boldsymbol{a}-\left(\mathbf{I}-e^{-\boldsymbol{\Omega}_{2} t}\right) \boldsymbol{b}+\boldsymbol{c},
$$

with I the identity matrix, $\boldsymbol{\Omega}_{i}=\operatorname{diag}\left(\omega_{1}^{(i)}, \ldots, \omega_{m}^{(i)}\right), i=$ 1,2 , and $\boldsymbol{a}, \boldsymbol{b}, \boldsymbol{c}$ constant vectors. The complex numbers $\omega_{1}^{(i)}, \ldots, \omega_{m}^{(i)}, i=1,2$, are called stiffness parameters. Assuming that $\mathbf{F}(t)$ coincides with $\boldsymbol{\Psi}(t)$ at $t_{n}$ and $t_{n+1}$, that $\mathbf{F}^{\prime}(t)$ coincides with $\boldsymbol{f}(t, \boldsymbol{\Psi})$ at $t_{n}$, and that $\mathbf{F}^{\prime \prime}(t)$ coincides with $\boldsymbol{f}^{\prime}(t, \boldsymbol{\Psi})$ at $t_{n}$, the solution $\boldsymbol{\Psi}_{n+1}=\boldsymbol{\Psi}\left(t_{n+1}\right)$ at $t_{n+1}$ can be expressed recursively in terms of $\boldsymbol{\Psi}_{n}=\boldsymbol{\Psi}\left(t_{n}\right), \boldsymbol{f}_{n}=$ $\boldsymbol{f}\left(t_{n}, \boldsymbol{\Psi}_{n}\right)$, and $\boldsymbol{f}_{n}^{(1)}=d \boldsymbol{f} /\left.d t\right|_{t=t_{n}}$ according to

$$
\boldsymbol{\Psi}_{n+1}=\boldsymbol{\Psi}_{n}+\mathbf{R} \boldsymbol{f}_{n}+\mathbf{S} \boldsymbol{f}_{n}^{(1)} .
$$

$\mathbf{R}$ and $\mathbf{S}$ are diagonal matrices which can be written in terms of the stiffness parameters:

$$
\mathbf{R}=\boldsymbol{\Omega}_{2} \boldsymbol{\Phi}-\boldsymbol{\Omega}_{1} \boldsymbol{\Xi}, \quad \boldsymbol{S}=\boldsymbol{\Phi}+\boldsymbol{\Xi},
$$

where $\boldsymbol{\Phi}$ and $\boldsymbol{\Xi}$ are diagonal matrices of which the nonzero entries are

$$
\Phi_{i}=\frac{e^{\omega_{i}^{(1)} h}-1}{\omega_{i}^{(1)}\left(\omega_{i}^{(1)}+\omega_{i}^{(2)}\right)}
$$

and

$$
\Xi_{i}=\frac{e^{-\omega_{i}^{(2)} h}-1}{\omega_{i}^{(2)}\left(\omega_{i}^{(1)}+\omega_{i}^{(2)}\right)} .
$$

Notice that if a stiffness parameter $\omega_{i}^{(1)}\left(\omega_{i}^{(2)}\right)$ vanishes the associated matrix element reads

$$
\Phi_{i}=\frac{h}{\omega_{i}^{(2)}} \quad\left(\Xi_{i}=-\frac{h}{\omega_{i}^{(1)}}\right) .
$$

The recursive relation (21) depends on the so far unknown stiffness parameters. These can be written in terms of the function $\boldsymbol{f}\left(t_{n}, \boldsymbol{\Psi}_{n}\right)$ and its time derivatives $\boldsymbol{f}_{n}^{(k)}, k=0,1,2,3$, at $t_{n}$ [31],

$$
\begin{gathered}
\omega_{i}^{(1)}=\frac{1}{2}\left[-D_{i}+\sqrt{D_{i}^{2}+4 E_{i}}\right] \\
\omega_{i}^{(2)}=\omega_{i}^{(1)}+D_{i},
\end{gathered}
$$

where $D_{i}$ and $E_{i}, i=1, \ldots, m$, are given in terms of the respective components $f_{i n}^{(k)}$ of $\boldsymbol{f}_{n}^{(k)}, k=0,1,2,3$, at $t=t_{n}$ by

$$
\begin{aligned}
& D_{i}=\frac{f_{i n}^{(0)} f_{i n}^{(3)}-f_{i n}^{(1)} f_{i n}^{(2)}}{f_{i n}^{(1)} f_{i n}^{(1)}-f_{i n}^{(0)} f_{i n}^{(2)}}, \quad i=1, \ldots, m, \\
& E_{i}=\frac{f_{i n}^{(1)} f_{i n}^{(3)}-f_{i n}^{(2)} f_{i n}^{(2)}}{f_{i n}^{(1)} f_{i n}^{(1)}-f_{i n}^{(0)} f_{i n}^{(2)}}, \quad i=1, \ldots, m,
\end{aligned}
$$

provided that the denominator of the previous expressions is nonzero. It is important to note that, in the present case, the time-dependent scaling function (9) is considered as a parameter. As a result, the successive time derivatives of this function are not taken into account in the calculation of $D_{i}$ and $E_{i}$.

The $i$ th component of the local truncation error at $t=t_{n+1}$, that is, the difference between the exact solution at $t_{n+1}$ and the numerical solution, is given by (we ignore here the index $i$ of the components for the sake of clarity) [31]

$$
\begin{aligned}
T_{n+1}= & \frac{h^{5}}{5 !} \frac{1}{\omega_{1}+\omega_{2}}\left[\left(\omega_{1}+\omega_{2}\right) f_{n}^{(4)}+\left(\omega_{2}^{4}-\omega_{1}^{4}\right) f_{n}^{(1)}\right. \\
& \left.-\left(\omega_{1}^{4} \omega_{2}+\omega_{1} \omega_{2}^{4}\right) f_{n}^{(0)}\right]+O\left(h^{6}\right) \\
= & \frac{h^{5}}{5 !}\left[f_{n}^{(4)}+\left(\omega_{2}^{3}-\omega_{2}^{2} \omega_{1}+\omega_{2} \omega_{1}^{2}-\omega_{1}^{3}\right) f_{n}^{(1)}\right. \\
& \left.-\omega_{1} \omega_{2}\left(\omega_{1}^{2}-\omega_{1} \omega_{2}+\omega_{2}^{2}\right) f_{n}^{(0)}\right]+O\left(h^{6}\right) .
\end{aligned}
$$

The implementation of the recursion (21) is now rather simple. It requires the calculation of the function $\boldsymbol{f}_{n}$ and its derivative $\boldsymbol{f}_{n}^{(1)}$ at each value of $t_{n}$. For the stiffness matrices $\boldsymbol{\Omega}_{1}$ and $\boldsymbol{\Omega}_{2}$, and thus also for the matrices $\mathbf{R}$ and $\mathbf{S}$, the derivatives $\boldsymbol{f}_{n}^{(2)}$ and $\boldsymbol{f}_{n}^{(3)}$ are also needed. $\boldsymbol{\Omega}_{1}$ and $\boldsymbol{\Omega}_{2}$ have to be calculated in principle at each integration step, since they characterize the local frequencies of the solution $\boldsymbol{\Psi}(t)$. The truncation error (29) can be used to control the size of the integration step, e.g., by imposing a boundary criterion for $\left|T_{n+1}\right|$. For this also, the derivative $\boldsymbol{f}_{n}^{(4)}$ must be provided.

The stiffness parameters carry the intrinsic information about the natural oscillations of the system. Therefore, the time step is expected to be rather large compared with standard explicit methods, like the Runge-Kutta method. In order to illustrate this, we solve without any time-dependent scaling of the coordinates the TDSE (18) for our one-dimensional model atom $\left(V_{0}=1\right.$ and $\left.\beta=1\right)$ interacting with a six-cycle cosine-squared pulse of $10^{13} \mathrm{~W} / \mathrm{cm}^{2}$ peak intensity and 0.7 a.u. photon energy (the same case as in Fig. 3). The time propagation is performed in the Hermite-Sturmian basis by means of two approaches: Fatunla's method and a fifth-order embedded Runge-Kutta formula [43]. The Hermite-Sturmian basis contains 500 functions with the dilation parameter $\alpha=0.5$ a.u. In Fig. 6 , we compare the time variations of the time step, which is automatically adjusted in both methods. In the case of Fatunla's method, this is done according to the condition $10^{-16} \leqslant T_{n+1} \leqslant 10^{-12}$ for the truncation error. In the case of the embedded Runge-Kutta formula, the time step is adjusted by comparing the results obtained with the fifth-order formula and with a fourth-order one which uses the same intermediate mesh points. We clearly see that the 


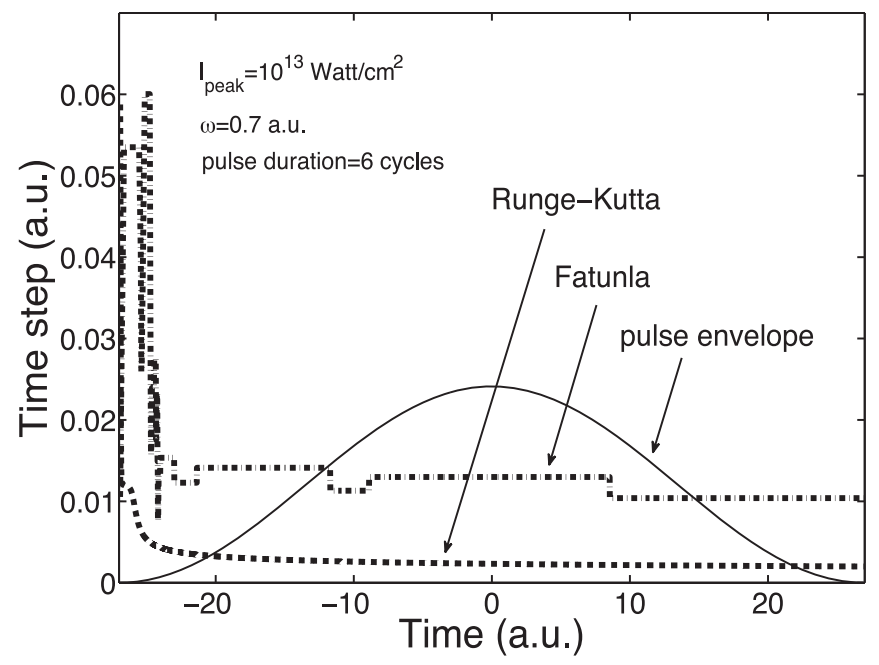

FIG. 6. Comparison of the time steps used in Fatunla's method (dashed line) and a method based on a fifth-order embedded RungeKutta formula (dotted line) to solve the TDSE for the same case as in Fig. 3. The time propagation is performed in a basis of 500 Hermite-Sturmian functions of dilation parameter $\alpha=0.5$ a.u. For the sake of completeness, we also show the pulse envelope.

time step is bigger in the case of Fatunla's method when compared to the Runge-Kutta embedded formula. However, the relative error on the conservation of the norm of the wave packet is, in this particular case, two orders of magnitude better in the case of the Runge-Kutta embedded formula. In

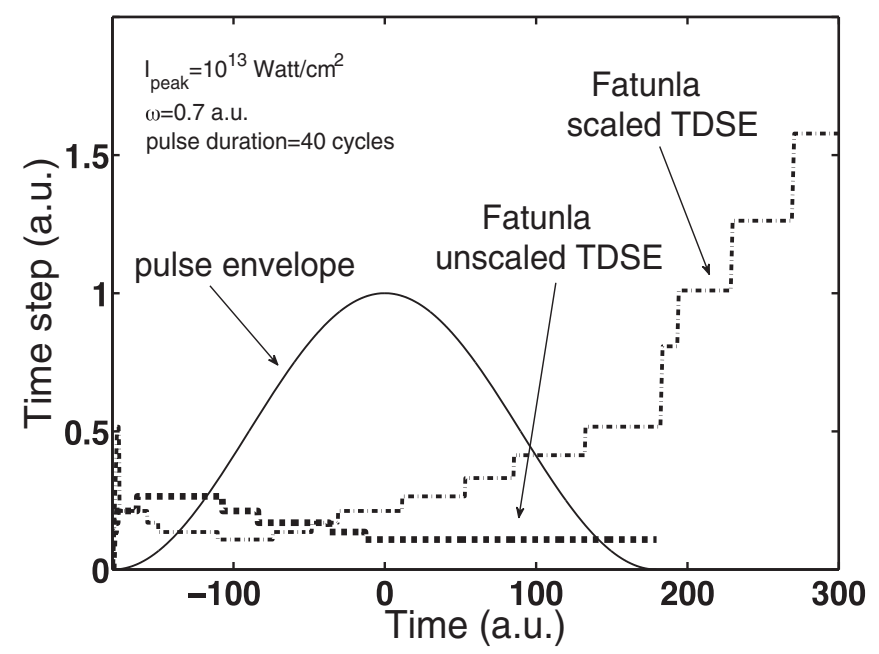

FIG. 7. Comparison of the time steps used in Fatunla's method to solve the scaled TDSE (light dotted line) and the unscaled TDSE (thick dotted line) in the case of the interaction of our one-dimensional model atom $\left(V_{0}=1\right.$ and $\left.\beta=1\right)$ with a cosine-squared pulse of $10^{13} \mathrm{~W} / \mathrm{cm}^{2}$ peak intensity and 0.7 a.u. photon energy. The total pulse duration is 40 cycles. The scaled and unscaled TDSEs are solved in a Hermite-Sturmian basis. In the case of the scaled TDSE, 400 Hermite-Sturmian functions are used. These functions have a dilation parameter $\alpha=0.05$ a.u. The parameter $n$ of the scaling function is equal to 4 and the asymptotic velocity $R_{\infty}=0.01$ a.u. For the unscaled TDSE, a basis of 2000 functions with a dilation parameter $\alpha=0.008$ a.u. is necessary. For the sake of completeness, we also show the pulse envelope. fact, by increasing the value of the dilation parameter $\alpha$ as well as the number of basis functions, the stiffness of the system of equations becomes more pronounced. In these conditions, Fatunla's method becomes much more efficient in terms of the magnitude of the time step and the relative error on the conservation of the norm is of the same order of that obtained with the Runge-Kutta embedded formula.

Fatunla's method has also been tested in much more demanding cases, namely, the interaction of atomic hydrogen with intense low-frequency pulses. The corresponding TDSE has been solved in a Coulomb-Sturmian basis. The results obtained with Fatunla's method are in very good agreement with those obtained with a fully implicit Radau method of order 7 (see Refs. [33,41] for details). In fact, Fatunla's method turned out to be ten times faster than the fully implicit method. However, the relative error on the conservation of the norm of the solution is hardly lower than $10^{-5}$, by contrast with the fully implicit method, where the norm is perfectly conserved.

Let us now examine how Fatunla's method performs in the case of the scaled TDSE. We consider the interaction of our one-dimensional model atom $\left(V_{0}=1\right.$ a.u. and $\beta=1$ a.u. $)$ with a cosine-squared pulse of $10^{13} \mathrm{~W} / \mathrm{cm}^{2}$ peak intensity and 0.7 a.u. photon energy. The total pulse duration is equal to 40 optical cycles. In Fig. 7, we show how the time step used by Fatunla's method varies with time for the scaled and unscaled TDSEs. We work in the Hermite-Sturmian basis. In the case of the scaled TDSE, 400 basis functions with a dilation parameter $\alpha=0.05$ a.u. are sufficient. The parameter $n$ of the scaling function is equal to 4 and the asymptotic velocity $R_{\infty}=0.01$ a.u. It is important to note that, in this case, it is necessary to integrate over about 4000 a.u. of time to reach the region where the scaled wave packet becomes static. By contrast with the unscaled equation, we clearly see that the time step needed for the time propagation of the scaled wave packet increases significantly as soon as the interaction with the pulse has ceased. In fact, around $t=4000$ a.u., the time step has a value around 10 a.u. This results from the subtraction of the scaled bound state at the end of the pulse and from the slow time evolution of the ionized wave packet in the scaled representation. It is important to stress here that the shrinking of the bound states will never stop, whereas the ionized part of the wave packet becomes static at large times. It is therefore important to subtract the scaled bound states from the total wave packet. Note that the subtraction has to be performed when both the interaction with the pulse and the harmonic potential have disappeared.

From the previous discussion, it is clear that Fatunla's method with adaptive step size is particularly well adapted to the solution of the scaled TDSE. In very stiff problems, the time step is much bigger than in the case of usual explicit Runge-Kutta methods. However, in all cases we have treated so far, the relative error on the conservation of the norm is of the order of $10^{-5}$, even when we force the time step to be very small. In many cases, this accuracy is sufficient but there are always cases where a higher accuracy is needed. In the next subsection, we show that this accuracy problem is solved by using a predictor-corrector scheme in which Fatunla's method is the predictor. 


\section{B. Predictor-corrector scheme}

Predictor-corrector (PC) methods are pairs of an explicit and an implicit multistep method where the explicit formula is used to predict the next approximation and the implicit formula to correct it. The order of the implicit method is usually the same as or higher than the order of the explicit method. In the present case, the predictor is Fatunla's method, which is of order 5. The corrector is a fully implicit four-stage Radau method of order 7 [34]. The implementation of this implicit method within the PC scheme follows Refs. [44] and [45]. It is based on diagonally implicit iterations and has two main advantages: it preserves the favorable stability characteristics of the fully implicit Radau method and it is suitable for use on parallel processors. In the following, we give a brief outline of the method. Let $t_{n}$ and $t_{n+1}$ be two consecutive times at which we want to calculate the wave packet. The solution $\boldsymbol{\Psi}_{n+1} \equiv \boldsymbol{\Psi}\left(t_{n+1}\right)$ of the TDSE is obtained from $\boldsymbol{\Psi}_{n}$ through the following relation:

$$
\boldsymbol{\Psi}_{n+1}=\boldsymbol{\Psi}_{n}+h \sum_{i=1}^{4} b_{i} \mathbf{H}\left(t_{n}+c_{i} h\right) \mathbf{Y}_{i}
$$

where $h=t_{n+1}-t_{n}$ and $b_{i}$ and $c_{i}$ are coefficients that define Radau's method. $t_{i}=t_{n}+c_{i} h$ with $i$ varying from 1 to 4 are intermediate times in the interval $\left[t_{n}, t_{n+1}\right]$ with $t_{4}=t_{n+1}\left(c_{4}=\right.$ 1). In the case of an orthonormal basis, $\mathbf{H}$ is the Hamiltonian matrix. When the basis is not orthonormal, $\mathbf{H}$ represents the Hamiltonian matrix multiplied on the left by the inverse of the overlap matrix. $\mathbf{Y}_{i}$ is an estimation of $\boldsymbol{\Psi}\left(t_{i}\right)$ which, within the fully implicit four-stage Radau method, is obtained by solving the following system:

$$
\left(\begin{array}{c}
\mathbf{Y}_{1} \\
\vdots \\
\mathbf{Y}_{4}
\end{array}\right)=\left(\begin{array}{c}
\Psi_{n} \\
\vdots \\
\Psi_{n}
\end{array}\right)+h\left(\begin{array}{ccc}
a_{11} \mathbf{H}\left(t_{1}\right) & \cdots & a_{14} \mathbf{H}\left(t_{4}\right) \\
\vdots & \ddots & \vdots \\
a_{41} \mathbf{H}\left(t_{1}\right) & \cdots & a_{44} \mathbf{H}\left(t_{4}\right)
\end{array}\right)\left(\begin{array}{c}
\mathbf{Y}_{1} \\
\vdots \\
\mathbf{Y}_{4}
\end{array}\right) \text {, }
$$

where the coefficients $a_{i j}$ are given. They define, like $b_{i}$ and $c_{i}$, the implicit four-stage Radau formula. If $\mathbf{H}$ is an $m \times m$ matrix, the dimension of the above system is $4 m$. Usually, when an implicit method is the corrector in a PC scheme, the vector that contains the $\mathbf{Y}_{i}$ in the right-hand side of Eq. (31) is provided by the predictor and the system (31) is solved iteratively. Those iterations are called explicit since they require only matrixvector multiplications. However, for stiff problems, such an explicit iterative process does not always converge. Instead, we introduce the diagonal matrix $\mathbf{D}=\operatorname{diag}\left(d_{11}, d_{22}, d_{33}, d_{44}\right)$ and rewrite system (31) as follows:

$$
\left(\begin{array}{c}
\mathbf{Y}_{1}^{(j)} \\
\vdots \\
\mathbf{Y}_{4}^{(j)}
\end{array}\right)-h\left(\begin{array}{ccc}
d_{11} \mathbf{H}\left(t_{1}\right) & \cdots & 0 \\
\vdots & \ddots & \vdots \\
0 & \cdots & d_{44} \mathbf{H}\left(t_{4}\right)
\end{array}\right)\left(\begin{array}{c}
\mathbf{Y}_{1}^{(j)} \\
\vdots \\
\mathbf{Y}_{4}^{(j)}
\end{array}\right)=\left(\begin{array}{c}
\boldsymbol{\Psi}_{n} \\
\vdots \\
\boldsymbol{\Psi}_{n}
\end{array}\right)+h\left(\begin{array}{ccc}
\left(a_{11}-d_{11}\right) \mathbf{H}\left(t_{1}\right) & \cdots & a_{14} \mathbf{H}\left(t_{4}\right) \\
\vdots & \ddots & \vdots \\
a_{41} \mathbf{H}\left(t_{1}\right) & \cdots & \left(a_{44}-d_{44}\right) \mathbf{H}\left(t_{4}\right)
\end{array}\right)\left(\begin{array}{c}
\mathbf{Y}_{1}^{(j-1)} \\
\vdots \\
\mathbf{Y}_{4}^{(j-1)}
\end{array}\right)
$$

where the superscript $j$ gives the order of the iteration. $j=$ 0 corresponds to the result obtained with Fatunla's method. Although systems (31) and (32) are perfectly equivalent since matrix $\mathbf{D}$ is subtracted on both sides of Eq. (31), we have now to solve four $m \times m$ systems of equations at each iteration. Those iterations are called implicit. Note that, due to the diagonal structure of matrix $\mathbf{D}$, the four systems can be solved in parallel. Such an implicit iterative process converges rapidly, requiring a few iterations. The choice of matrix $\mathbf{D}$ is in principle arbitrary. In the present case, it is calculated in order to preserve the stability properties of the fully implicit Radau method. See Ref. [44] for more details. The present PC method allows a perfect conservation of the norm. Furthermore, we checked that the four systems of equations can be easily solved by means of the biconjugate gradient algorithm which requires only matrix-vector multiplications. Therefore, although the present PC algorithm is of an implicit nature, it is easy to implement on parallel processors.

\section{ELECTRON ENERGY SPECTRUM}

One of the main advantages of the TSC method is the fact that the electron energy spectrum may be expressed directly in term of the scaled wave packet at large times. Here, we show it explicitly in the case of our one-dimensional model and for atomic hydrogen.

\section{A. Analytical expression for the electron energy spectra}

\section{One-dimensional model}

The unscaled wave packet that is the solution of the TDSE (1) can always be written as follows:

$$
\Psi(x, t)=\sum_{n} a_{n}(t) \psi_{n}(x) e^{-i E_{n} t}+\int_{-\infty}^{\infty} c_{k}(t) \psi_{k}(x) e^{-i\left(k^{2} / 2\right) t} d k,
$$

where $\psi_{n}(x)$ is the wave function associated with a bound state of our model atom with $E_{n}$ its energy. $a_{n}(t)$ represents the probability amplitude for the system to be in the corresponding bound state. $\psi_{k}(x)$ is the wave function associated with a continuum state of energy $E=k^{2} / 2$ while $c_{k}(t)$ is the corresponding probability amplitude. $k$ is the wave vector: a positive value of $k$ corresponds to a wave propagating to the right while a negative value corresponds to a propagation to 
the left. The continuum wave functions satisfy the following orthogonality relation:

$$
\int_{-\infty}^{\infty} \psi_{k}^{*}(x) \psi_{k^{\prime}}(x) d x=\delta\left(k-k^{\prime}\right) .
$$

In order to calculate the electron energy spectrum given by $\left|c_{k}(t \rightarrow \infty)\right|^{2}$, we let $x \rightarrow \pm \infty$ in Eq. (33). In this limit the first term of the right-hand side of this equation goes to zero and

$$
\psi_{k}(x \rightarrow \infty)=\frac{1}{\sqrt{2 \pi}} e^{i k x} .
$$

Note that the limit $x \rightarrow-\infty$ leads to the same expression since $k$ is replaced by $-k$. As a result we have

$$
\Psi(x \rightarrow \pm \infty, t)=\frac{1}{\sqrt{2 \pi}} \int_{-\infty}^{\infty} c_{k}(t) e^{i t\left[(k / t) x-k^{2} / 2\right]} d k .
$$

In order to calculate the spectrum, let us take the limit of the above integral for $t \rightarrow \infty$. By using the stationary phase theorem, we obtain

$$
\Psi(x \rightarrow \pm \infty, t \rightarrow \infty)=c_{k}(t \rightarrow \infty) \frac{1}{\sqrt{i t}} e^{i x^{2} / 2 t},
$$

where $k=x / t$ is the stationary phase point. From Eqs. (6) and (9), we write

$$
k=\frac{x}{t}=R_{\infty}\left(1-\frac{t_{\mathrm{sc}}}{t}\right) \xi .
$$

Using Eq. (7) that relates the scaled wave packet to the unscaled one, we finally get

$$
c_{k}(t \rightarrow \infty)=\sqrt{i\left(\frac{1}{R_{\infty}}+\frac{t_{\mathrm{sc}}}{R(t)}\right)} \Phi\left(\frac{k}{R_{\infty}\left(1-\frac{t_{\mathrm{sc}}}{t}\right)}, t \rightarrow \infty\right),
$$

which establishes a direct link between the probability amplitude for the electron to be in the continuum with an energy $k^{2} / 2$ and the scaled wave packet at large times. Below, we discuss results for the electron energy spectrum and compare them with those obtained by solving the unscaled TDSE. In this latter case, we calculate the energy spectrum by projecting the final wave packet onto continuum pseudostates obtained by diagonalizing the atomic Hamiltonian. Note that we get the same result by projecting onto plane waves because the phase shift introduced by the short-range Gaussian potential is negligible.

\section{Atomic hydrogen}

As in the previous case, the unscaled wave packet can be written as follows:

$$
\begin{aligned}
\Psi(\vec{r}, t)= & \sum_{n, l, m} a_{n, l}(t) \psi_{n, l}(r) Y_{l, m}(\theta, \phi) e^{-i E_{n} t} \\
& +\sum_{l, m} \int_{0}^{\infty} c_{l k}(t) R_{l}(k r) Y_{l, m}(\theta, \phi) e^{-i\left(k^{2} / 2\right) t} k d k,
\end{aligned}
$$

where $\psi_{n, l}(r)$ is the radial wave function of a hydrogen bound state of energy $E_{n}$ and $a_{n, l}$ the corresponding probability amplitude. $Y_{l, m}(\theta, \phi)$ is a spherical harmonic that is a function of the angular coordinates of vector $\vec{r} . R_{l}(k r)$ is the radial regular Coulomb function where $k$ is the magnitude of the electron momentum [46]. $c_{l k}(t)$ is the probability amplitude for the electron to be in the continuum. The asymptotic form of the radial regular Coulomb function is

$$
R_{l}(k r) \underset{r \rightarrow \infty}{\rightarrow} N_{l}(k) \frac{1}{k r} \sin \left(k r-\frac{1}{2} l \pi+\frac{1}{k} \ln (2 k r)+\sigma_{l}\right) .
$$

$N_{l}(k)$ is a normalization factor. In the present case, it is equal to $2 \sqrt{k}$ because $R_{l}(k r)$ is normalized per unit energy. $\sigma_{l}$ is the Coulomb phase shift given by

$$
\sigma_{l}=\arg \Gamma\left(l+1-\frac{i}{k}\right),
$$

where $\Gamma(x)$ is the Gamma function. In the limit of large distances, the unscaled wave packet reduces to the following expression:

$$
\begin{aligned}
\Psi(\vec{r}, t) \underset{r \rightarrow \infty}{\rightarrow} & \sum_{l, m} \int_{0}^{\infty} \frac{\sqrt{k}}{i r} c_{l k}(t) e^{-i\left(k^{2} / 2\right) t} \\
& \times\left[e^{i\left[k r-l \pi / 2+\sigma_{l}+(1 / k) \ln (2 k r)\right]}\right. \\
& \left.-e^{-i\left[k r-l \pi / 2+\sigma_{l}+(1 / k) \ln (2 k r)\right]}\right] d k .
\end{aligned}
$$

Note that the sine function in the asymptotic expression (41) of the regular Coulomb function has been replaced by a sum of two complex exponentials that describe pure ingoing and outgoing spherical waves at large distances. In order to calculate the electron energy spectrum, we now examine the limit of the previous expression for $t \rightarrow \infty$. Before applying the stationary phase theorem, let us rewrite expression (43) as follows:

$$
\begin{aligned}
\Psi(\vec{r}, t) \underset{r \rightarrow \infty}{\rightarrow} & \sum_{l, m} \int_{0}^{\infty} \frac{\sqrt{k}}{i r} c_{l k}(t) e^{i\left(\sigma_{l}+l \pi / 2\right)} \\
& \times e^{i t\left[-k^{2} / 2+k r / t+(1 / k t) \ln (2 k r)\right]} d k .
\end{aligned}
$$

In fact, it is easy to show that the ingoing spherical wave present in expression (43) does not contribute to the integral in the limit $t \rightarrow \infty$. In integral (44), the stationary phase point $k=k_{0}$ is the solution of the following equation:

$$
-k+\frac{r}{t}-\frac{1-\ln (2 k r)}{k^{2} t}=0 .
$$

To a good approximation we can replace $k$ by $r / t$ in the third term of the left-hand side of the above equation. As a result we obtain

$$
k_{0}=\frac{r}{t}-\frac{1-\ln \left(2 \frac{r^{2}}{t}\right)}{\frac{r^{2}}{t}} .
$$

If $k_{e}$ represents the velocity of the electron at large distances, we can show by means of classical mechanics that for large times

$$
\frac{r}{t} \approx k_{e}+\frac{1}{k_{e}^{2} t} \ln \left(1+k_{e}^{3} t\right) .
$$




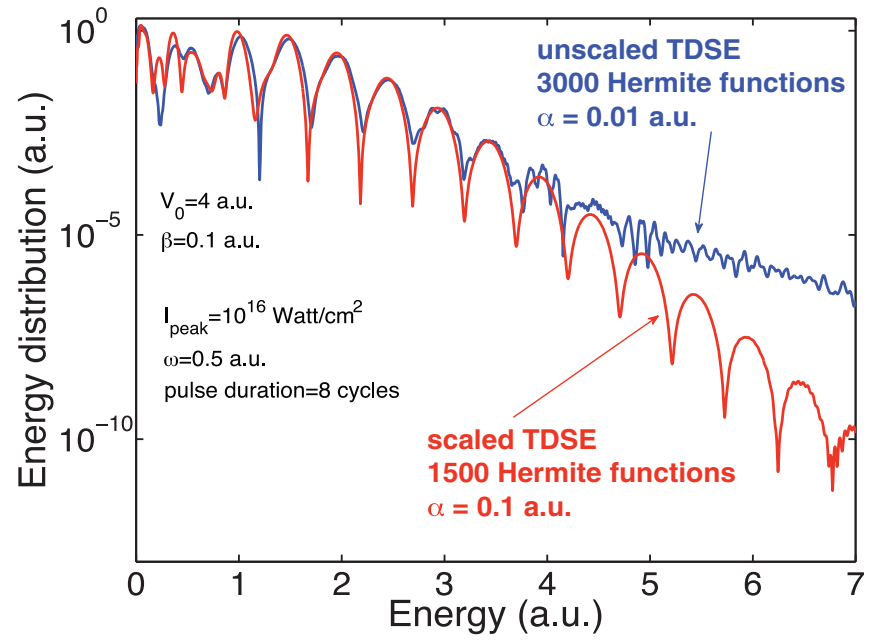

FIG. 8. (Color online) Electron energy spectrum resulting from the interaction of our model atom with a strong low-frequency pulse. The Gaussian potential parameters $V_{0}=4$ a.u. and $\beta=0.1$ a.u. are such that this potential supports seven bound states. The cosinesquared pulse of peak intensity $10^{16} \mathrm{~W} / \mathrm{cm}^{2}$ and frequency $\omega=$ 0.5 a.u. has a total duration of eight optical cycles. The full blue line is the result obtained by solving the unscaled TDSE with a basis of 3000 Hermite-Sturmian functions and a dilation parameter $\alpha=0.01$ a.u. The full red line is the result obtained by solving the scaled TDSE with a basis of 1500 Hermite-Sturmian functions and a dilation parameter $\alpha=0.1$ a.u.

Note that for large times, $k_{0}$, the stationary phase point, coincides with $k_{e}$. By using the stationary phase theorem and Eqs. (6), (7), and (9), we obtain

$$
\begin{aligned}
c_{l k_{0}}(t \rightarrow \infty)= & {\left[i\left(\frac{1}{R_{\infty}}+\frac{t_{\mathrm{sc}}}{R(t)}\right)\right]^{-3 / 2}\left(\frac{r}{t}\right) \frac{e^{-i\left(\sigma_{l}-\pi / 2\right)}}{\sqrt{k_{0}}} } \\
& \times \exp \left[i\left(\frac{\ln \left(2 r^{2} / t\right)+2 \frac{\ln \left(2 r^{2} / t\right)}{r^{2} / t}}{\frac{r}{t}+\frac{\ln \left(2 r^{2} / t\right)}{r^{2} / t}}\right)\right] \\
& \times \Phi_{l}\left(\frac{r}{R_{\infty}\left(t-t_{\mathrm{sc}}\right)}, t \rightarrow \infty\right)
\end{aligned}
$$

which for a given value of the angular momentum $l$ establishes a link between the probability amplitude for the electron to be in the continuum with an energy $E=k_{e}^{2} / 2 \approx k_{0}^{2} / 2$ and the corresponding $l$ component of the scaled wave packet and hence the electron energy spectrum. Our results for the energy spectrum are compared with those obtained by projecting the unscaled wave packet onto Coulomb functions.

\section{B. Results and discussion}

As a proof of principle, we show in this section that the present method provides very accurate results for the electron energy spectra at the expense of fewer computer resources than with the same propagation method without scaling. Here, we calculate two different electron energy spectra in rather demanding physical situations. First, we consider the ionization of our one-dimensional model atom with an intense low-frequency field.

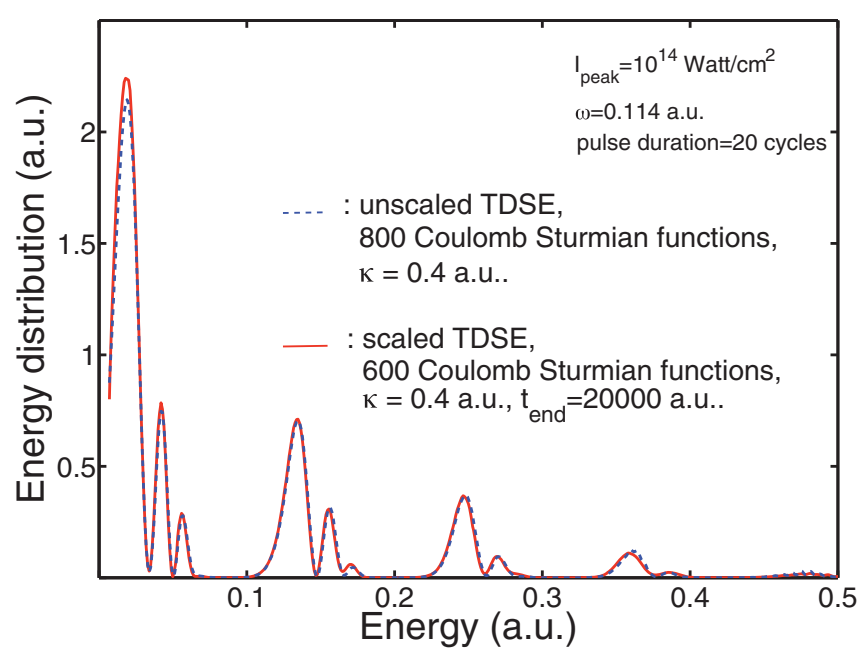

FIG. 9. (Color online) Electron energy spectrum resulting from the interaction of atomic hydrogen with a strong low-frequency laser field. The cosine-squared pulse of peak intensity $10^{14} \mathrm{~W} / \mathrm{cm}^{2}$ and frequency $\omega=0.114$ a.u. has a total duration of 20 optical cycles. The blue dashed line is the result obtained by solving the unscaled TDSE with a basis of 800 Coulomb Sturmians per electron angular momentum and a dilation parameter $\kappa=0.4$ a.u. The full red line is the result obtained by solving the scaled TDSE with a basis of 600 Coulomb-Sturmian functions per electron angular momentum and the same dilation parameter. The scaled wave packet is propagated until $t_{\text {end }}=20000$ a.u. of time, where it is stationary.

The Gaussian potential parameters ( $V_{0}=4$ a.u., and $\beta=$ 0.1 a.u.) are such that it supports seven bound states, the ground-state energy being equal to -3.572 a.u. The cosinesquared pulse has a total duration of eight optical cycles with a peak intensity of $10^{16} \mathrm{~W} / \mathrm{cm}^{2}$ and a frequency of 0.5 a.u. The time propagation of both the unscaled and scaled wave packets has been performed by means of the predictor-corrector method described above. The unscaled TDSE has been solved by expanding the wave packet in a basis of 3000 Hermite-Sturmian functions with a dilation parameter $\alpha=0.01$ a.u. The result is shown in Fig. 8 (the full blue line). We clearly see that the energy spectrum becomes noisy already around an electron energy of 3 a.u. The reason is the following. Since fast electrons are emitted, the problem of the reflection of the unscaled wave packet by the numerical boundaries is a crucial issue. In order to overcome this problem, it is necessary to use a small value of the dilation parameter. In that case, however, the density of positive-energy states we obtain by diagonalizing the atomic Hamiltonian gets very small at high electron energy, thereby leading to the noisy behavior of the energy spectrum above 3 a.u. The scaled TDSE has been solved by using a smaller basis of 1500 Hermite-Sturmian functions with a dilation parameter of $\alpha=0.1$ a.u. The correct spectrum (red full line) is obtained over more than ten orders of magnitude after the scaled wave packet has been time propagated over 5000 a.u. of time. The origin of the discrepancies observed at low electron energies is related to the choice of the asymptotic velocity. In the present case, $R_{\infty}=0.05$ a.u. This value is in fact too high and leads to a strong confinement and thereby to a poor description of the shrinking of the bound states 
during the interaction with the pulse. When $R_{\infty}=0.03$ a.u., the discrepancies disappear and the two curves are in good agreement at low electron energies. However, if the size of the basis stays equal to 1500 , the result obtained by solving the scaled TDSE exhibits some unphysical oscillations at high energy.

In that case, the confinement is weaker, requiring an increase of the size of the basis to cover a more extended region of space. An alternative could be to decrease the value of the dilation parameter. However, this dilation parameter fixes the spatial resolution in the whole space covered by the basis. It is therefore clear that a multiresolution technique in which the resolution is increased locally and gradually around the origin is more appropriate.

In Fig. 9, we consider the case of atomic hydrogen interacting with a 20-optical-cycle cosine-squared pulse of peak intensity $10^{14} \mathrm{~W} / \mathrm{cm}^{2}$ and frequency 0.114 a.u. The blue dashed line is the electron spectrum obtained by solving the unscaled TDSE with a basis of 800 Coulomb-Sturmian functions with a dilation parameter $\kappa$ equal to 0.4 a.u. This result is in perfect agreement with the one obtained by Grum-Grzhimailo et al. [47] (see Fig. 4 of that reference). The full red line is the electron spectrum obtained by solving the scaled TDSE with a basis of 600 Coulomb-Sturmian functions with the same dilation parameter. Note that we have to propagate the scaled wave packet until $t_{\mathrm{end}}=20000$ a.u., i.e., for a long time after the end of the pulse. In fact, we have to wait until the wave packet becomes stationary before calculating the energy spectrum. In this context, it is therefore crucial to extract the scaled bound states. We clearly see that the results obtained by time-scaling the coordinates are in perfect agreement with those obtained without scaling except at very small electron energies (the first peak), where we observe a tiny difference, which, as before, is due to a slightly inaccurate description of the shrinking of the scaled bound states. In the present case, the scaling is switched on right at the beginning of the interaction and we use a small value of the asymptotic velocity, $R_{\infty}=0.001$ a.u., because of the long duration of the pulse.

\section{CONCLUSIONS AND PERSPECTIVES}

In this paper, we develop an ab initio approach to solve numerically the time-dependent Schrödinger equation that governs the ionization dynamics of atoms and molecules interacting with pulsed radiation fields. The approach is based on the combination of the time-scaled coordinate method with an efficient time propagator. The key points of the time-scaled coordinate method are a time-dependent scaling of the electron radial coordinate together with a phase transformation of the total wave packet of the system. This method presents the following advantages: (i) the fast oscillations resulting from the rapidly growing phase gradients are removed from the total wave packet thanks to the phase transformation, (ii) the scaled wave packet stays spatially confined while reaching a stationary state a sufficiently long time after the interaction with the pulse, and (iii) the electron energy distribution is proportional to the squared modulus of the scaled wave packet once it becomes stationary. This method has, however an important drawback: it introduces different length scales into the problem. In particular, it leads to a shrinking of the scaled bound states. In principle, such an effect can be described properly by using a denser grid or a much bigger basis of $\mathcal{L}^{2}$ functions. However, this inevitably increases the stiffness of the system of first-order differential equations that must be solved for the time propagation of the scaled wave packet. Here, we show one efficient way of treating these problems. First, it is important to subtract the scaled bound states from the total wave packet after the end of the pulse, once the harmonic potential that confines the wave packet has disappeared. Second, we introduce a highorder time propagator of predictor-corrector type that has so far been shown to be very efficient in handling the stiffness problem. The predictor is the fifth-order explicit method of Fatunla and the corrector a fully implicit Radau method of order 7. Despite the implicit character of the corrector, we show that all the calculations reduce to simple matrix-vector products that allow a high level parallelization of the computer codes. Finally, our calculations suggest that an elegant way to further improve the efficiency of our method is the use of multiresolution techniques.

At this stage, the method has been tested in the case of the interaction of a pulsed radiation field with a one-dimensional model atom described by a Gaussian potential and with atomic hydrogen. Electron energy spectra have been calculated in rather demanding physical situations. In all cases, our approach gives very accurate results, particularly for high photoelectron energies, at the expense of fewer computer resources when compared to the usual grid or spectral methods without scaling.

\section{ACKNOWLEDGMENTS}

The authors enjoyed very interesting discussions with Laurence Malegat and Yuri Popov. J.E., J.M., and P.O'M thank the Université Catholique de Louvain (UCL) for financially supporting several stays at the Institute of Condensed Mater and Nanosciences (IMCN) of the UCL. J.E. gratefully acknowledges financial support by Deutsche Forschungsgemeinschaft under Contract No. FR 591/16-1 and the Emmy-Noether Grant No. KR 2889/2. J.M. thanks the Deutsche Forschungsgemeinschaft for financial support under Contracts No. FR 591/16-1 and No. MA 3305/2-2. B.P. thanks Royal Holloway College, University of London, for hospitality and financial support. A.L.F. gratefully acknowledges the financial support of the IISN (Institut Interuniversitaire des Sciences Nucléaires) through Contract No 4.4.503.02.F, "Atoms, ions and radiation. Experimental and theoretical study of fundamental mechanisms governing laser-atom interactions and of radiative and collisional processes of astrophysical and thermonuclear relevance." The authors thank UCL for providing them with access to the supercomputer of the CISM (Calcul Intensif et Stockage de Masse) which is supported by the FNRS (Fonds National de la Recherche Scientifique) through the FRFC (Fonds de la Recherche Fondamentale Collective) Project No. 2.4556.99, "Simulations Numériques et traitement des données." 
[1] P. M. Paul, E. S. Toma, P. Breger, G. Mullot, F. Augé, Ph. Balcou, H. G. Muller, and P. Agostini, Science 292, 1689 (2001).

[2] J. Andruszkow et al., Phys. Rev. Lett. 85, 3825 (2000).

[3] E. S. Smyth, J. S. Parker, and K. T. Taylor, Comput. Phys. Commun. 114, 1 (1998).

[4] J. S. Parker, B. J. S. Doherty, K. T. Taylor, K. D. Schultz, C. I. Blaga, and L. F. Di Mauro, Phys. Rev. Lett. 96, 133001 (2006).

[5] A. Palacios, T. N. Rescigno, and C. W. McCurdy, Phys. Rev. A 79, 033402 (2009).

[6] L. Malegat, H. Bachau, A. Hamido, and B. Piraux, J. Phys. B 43, 245601 (2010).

[7] J. Colgan, M. S. Pindzola, and F. Robicheaux, J. Phys. B 34, L457 (2001).

[8] J. Feist, S. Nagele, R. Pazourek, E. Persson, B. I. Schneider, L. A. Collins, and J. Burgdörfer, Phys. Rev. A 77, 043420 (2008).

[9] X. Guan, K. Bartschat, and B. I. Schneider, Phys. Rev. A 77, 043421 (2008).

[10] X. Guan, O. Zatsarinny, C. J. Noble, K. Bartschat, and B. I. Schneider, J. Phys. B 42, 134015 (2009).

[11] I. A. Ivanov and A. Kheifets, J. Phys. B 41, 095002 (2008).

[12] E. Foumouo, G. L. Kamta, G. Edah, and B. Piraux, Phys. Rev. A 74, 063409 (2006).

[13] M. A. Lysaght, P. G. Burke, and H. W. van der Hart, Phys. Rev. Lett. 101, 253001 (2008).

[14] M. A. Lysaght, H. W. van der Hart, and P. G. Burke, Phys. Rev. A 79, 053411 (2009).

[15] J. R. Burgan, M. R. Feix, E. Fijalkow, and A. Munier, Phys. Lett. A 74, 11 (1979).

[16] G. Manfredi, S. Mola, and M. R. Feix, Phys. Fluids B 5, 388 (1993).

[17] S. Mola, G. Manfredi, and M. R. Feix, J. Plasma Phys. 80, 145 (1993).

[18] E. A. Solov'ev and S. I. Vinitsky, J. Phys. B 18, L557 (1985).

[19] S. Yu. Ovchinnikov, G. N. Ogurtsov, J. H. Macek, and Yu. S. Gordeev, Phys. Rep. 389, 119 (2004).

[20] E. Y. Sidky and B. D. Esry, Phys. Rev. Lett. 85, 5086 (2000).

[21] V. L. Derbov, M. S. Kaschiev, V. V. Serov, A. A. Gusev, and S. I. Vinitsky, Proc. SPIE 5067, 218 (2003).

[22] V. V. Serov, V. L. Derbov, B. B. Joulakian, and S. I. Vinitsky, Phys. Rev. A 63, 062711 (2001).

[23] V. V. Serov, V. L. Derbov, B. B. Joulakian, and S. I. Vinitsky, Phys. Rev. A 75, 012715 (2007).

[24] V. V. Serov, V. L. Derbov, B. B. Joulakian, and S. I. Vinitsky, Phys. Rev. A 78, 063403 (2008).

[25] E. Falize et al., J. Phys.: Conf. Ser. 112, 042015 (2008).
[26] Y. Castin and R. Dum, Phys. Rev. Lett. 77, 5315 (1996).

[27] L. Pitaevskii and S. Stringari, Bose-Einstein Condensation, (Oxford University Press, Oxford, 2004), Chap. 12, Sec. 7.

[28] V. Roudnev and B. D. Esry, Phys. Rev. A 71, 013411 (2005).

[29] J. D. Lambert, Numerical Methods for Ordinary Differential Systems: The Initial Value Problem (John Wiley and Sons, Chichester, 1991), Chap. 6.

[30] S. O. Fatunla, Math. Comput. 32, 1 (1978).

[31] S. O. Fatunla, Math. Comput. 34, 373 (1980).

[32] J. Madroñero and B. Piraux, Phys. Rev. A 80, 033409 (2009).

[33] J. Madroñero and B. Piraux, J. Phys.: Conf. Ser. 212, 012027 (2010).

[34] J. C. Butcher, Math. Comput. 18, 233 (1964).

[35] I. Daubechies, Ten Lectures on Wavelets (SIAM, Philadelphia, 1992).

[36] J. Eiglsperger, B. Piraux, and J. Madroñero, Phys. Rev. A 80, 022511 (2009).

[37] J. Eiglsperger, B. Piraux, and J. Madroñero, Phys. Rev. A 81, 042527 (2010).

[38] J. Eiglsperger, B. Piraux, and J. Madroñero, Phys. Rev. A 81, 042528 (2010).

[39] J. Eiglsperger, M. Schönwetter, B. Piraux, and J. Madroñero, e-print arXiv:1105.0742v1, At. Data Nucl. Data Tables (to be published).

[40] H. Bachau, E. Cormier, P. Decleva, J. E. Hansen, and F. Martín, Rep. Prog. Phys. 64, 1815 (2001).

[41] E. Huens, B. Piraux, A. Bugacov, and M. Gajda, Phys. Rev. A 55, 2132 (1997).

[42] L. Lapidus and J. H. Seinfeld, Numerical Solution of Ordinary Differential Equations (Academic Press, New York, 1971), Chap. 6.

[43] E. Hairer, S. P. Norsett, and G. Wanner, Solving Ordinary Differential Equations I: Non-Stiff Problems (Springer-Verlag, Berlin, 1987).

[44] P. J. van der Houwen and B. P. Sommeijer, SIAM J. Sci. Stat. Comput. 12, 1000 (1991).

[45] P. J. van der Houwen and B. P. Sommeijer, Appl. Numer. Math. 11, 169 (1993).

[46] N. F. Mott and H. S. W. Massey, The Theory of Atomic Collisions (Clarendon, Oxford, 1965), Chap. 21, Sec. 2.

[47] A. N. Grum-Grzhimailo, B. Abeln, K. Bartschat, D. Weflen, and T. Urness, Phys. Rev. A 81, 043408 (2010). 